\title{
ORIGINAL ARTICLE SATB1 collaborates with loss of p16 in cellular transformation
}

\author{
R Agrelo ${ }^{1,2}$, H Kishimoto ${ }^{2,3,4}$, M Novatchkova ${ }^{2}$, V Peraza ${ }^{1}$, M Paolino ${ }^{2}$, A Souabni ${ }^{2}$ and A Wutz ${ }^{2,4,5}$
}

Tumor progression is associated with invasiveness and metastatic potential. The special AT-rich binding protein 1 (SATB1) has been identified as a key factor in the progression of breast cancer cells to a malignant phenotype and is associated with progression of human tumors. In normal development, SATB1 coordinates gene expression of progenitor cells by functioning as a genome organizer. In contrast to progenitor and tumor cells, SATB1 expression in nontransformed cells is not compatible with proliferation. Here we show that SATB1 expression in mouse embryonic fibroblasts induces cell cycle arrest and senescence that is associated with elevated p16 protein levels. Deletion of p16 overcomes the SATB1-induced senescence. We further provide evidence for an interaction of SATB1 with the retinoblastoma (RB)/E2F pathway downstream of p16. A combined deletion of the RB proteins, RB, p107 and p130 (triple-mutant; TM), prevents SATB1-induced G1 arrest, which is restored upon the reintroduction of RB into SATB1-expressing TM fibroblasts. SATB1 interacts with the E2F/RB complex and regulates the cyclin E promoter in an E2F-dependent manner. These findings demonstrate that $\mathrm{p} 16$ and the RB/E2F pathway are critical for SATB1-induced cell cycle arrest. In the absence of p16, SATB1 causes anchorage-independent growth and invasive phenotype in fibroblasts. Our data illustrate that p16 mutations collaborate with the oncogenic activity of SATB1. Consistent with our finding, a literature survey shows that deletion of p16 is generally associated with SATB1 expressing human cell lines and tumors.

Oncogene (2013) 32, 5492-5500; doi:10.1038/onc.2013.158; published online 20 May 2013

Keywords: senescence; SATB1; p16; tumor; cell cycle; RB; E2F

\section{INTRODUCTION}

The special AT-rich binding protein 1 (SATB1) interacts with AT-rich sequences and facilitates the organization of chromatin., ${ }^{1,2}$ SATB1 is required for the coordinate regulation of gene expression in T-lymphocyte development. ${ }^{3}$ Mutation of SATB1 in mice leads to misregulation of a large number of T-cell-specific genes and causes defects in the thymocyte development. In addition, roles in stem cell proliferation and differentiation have been proposed. ${ }^{4,5}$ In T cells SATB1 shows ring-like nuclear distribution surrounding heterochromatic regions, ${ }^{6}$ whereby SATB1-bound sequences overlap the base of chromatin loops. ${ }^{6,7}$ It is thought that SATB1 contributes to the regulation of gene clusters by organization of chromatin conformation. ${ }^{6}$ A topological function in gene regulation is consistent with recent observations that SATB1 contributes to chromosome-wide gene repression during $\mathrm{X}$ inactivation. ${ }^{8}$

SATB1 interacts with chromatin-modifying complexes and regulates histone modifications and nucleosome positioning over large regions. ${ }^{7,9}$ Furthermore, SATB1 itself is subject to post-translational modifications that influence its activity. ${ }^{10-12}$ Phosphorylation-dependent interaction of SATB1 and PIAS1 directs SUMO-regulated caspase cleavage of SATB1. ${ }^{12}$ An acetylation-dependent interaction with C-terminal-binding protein 1 has also been implicated in the gene repression mechanism of SATB1. ${ }^{11}$ In T helper type-2 cell development, SATB1 interacts with $\beta$-catenin to activate cell type-specific target genes in a WNT-dependent manner. ${ }^{13}$ Foxp3 has recently been shown to repress SATB1 during the development of regulatory T cells. ${ }^{14}$ Release of SATB1 repression causes a loss of regulatory
T-cell identity and induces an effector T-cell program. ${ }^{14}$ SATB1 is further expressed in the basal cells of the epidermis where it is regulated by $063 .{ }^{15}$ Mutation of SATB1 causes gene expression changes in skin progenitor cells and impaired epidermal morphology. ${ }^{15}$ These findings show that SATB1 regulates cell type-specific gene expression in development.

SATB1 is associated with highly metastatic breast cancer. ${ }^{16}$ SATB1 expression in breast epithelial cell lines has been shown to induce a tumor-like expression profile and a malignant phenotype. ${ }^{16}$ This observation has led to the proposal that SATB1 expression outside its normal developmental context can reprogram cancer cells to a highly metastatic phenotype. SATB1 expression is also associated with other human tumors including rectal cancer and cutaneous malignant melanoma. ${ }^{17-21}$ Recently, the tumor suppressor FOXP3 has been shown to function at least in part by repressing SATB1 in breast and prostate epithelia. ${ }^{22}$ Conversely, interference with the chromatin association of SATB1 by pharmacological inhibition has been proposed as a potential therapeutic strategy. Initial result suggests a potential for reducing invasive and metastatic characteristics of certain human breast cancer cells. ${ }^{23}$ An independent prognostic value of SATB1 expression for breast cancer is currently debated. $^{24-26}$ These findings suggest that SATB1 is a regulator of tumor progression and metastatic potential. The effect of SATB1 expression in normal pretumorigenic cells has remained largely unclear. Here, we study the ability of SATB1 to induce cellular transformation and demonstrate the impact of key cell cyclerelated pathways.

${ }^{1}$ Institut Pasteur de Montevideo, Epigenetics of Cancer and Aging Laboratory, Montevideo, Uruguay; ${ }^{2}$ Research Institute of Molecular Pathology, Vienna, Austria; ${ }^{3}$ Life Science Center of Tsukuba Advanced Research Alliance, University of Tsukuba, Tsukuba-shi, Japan; ${ }^{4}$ Department of Biochemistry, Wellcome Trust and Medical Research Council Stem Cell Institute, University of Cambridge, Cambridge, UK and ${ }^{5}$ Institute of Molecular Health Sciences, Swiss Federal Institute of Technology, Zurich, Switzerland. Correspondence: Dr R Agrelo, Institut Pasteur de Montevideo, Epigenetics of Cancer and Aging Laboratory, Mataojo 2020, Montevideo, CP 11400, Uruguay.

E-mail: agrelo@pasteur.edu.uy

or Dr A Wutz, D-BIOL, ETH Zurich, Institute of Molecular Health Sciences, Swiss Federal Institute of Technology, Schafmattstrasse 22, Zurich 8049, Switzerland E-mail: awutz@ethz.ch

Received 27 September 2012; revised 11 March 2013; accepted 11 March 2013; published online 20 May 2013 


\section{RESULTS}

SATB1 expression induces senescence in MEFs

To understand the effects of SATB1 expression in cells better, we have used mouse embryonic fibroblasts (MEFs) as a tractable model for cellular transformation. We infected primary MEFs with viral vectors for SATB1 expression (pBABE-GFP-SATB1) or a control empty vector ( $\mathrm{PBABE}-\mathrm{GFP}-\mathrm{EV}$ ) and selected stable virus integrations with puromycin. Numerous colonies emerged in control but not in SATB1 virus-infected cultures. Only in one out of ten experiments, three colonies were recovered that expressed minute amounts of SATB1. As primary MEFs are limited to a certain passage in culture, we continued with MEFs that were immortalized but not transformed, using the 3 T3 protocol. After infection and puromycin selection for 7 days, highly expressing clones were isolated using an internal ribosomal entry site-linked green fluorescent protein (GFP) marker. MEFs expressing SATB1 grew markedly slower than controls (Figure 1a), stained positive for $\beta$-galactosidase activity and had a flat morphology (Figures $1 \mathrm{~b}$ and c), indicating a large fraction of SATB1-expressing cells displayed signs of senescence. Consistent with this, we observed a large $\mathrm{G} 1 / \mathrm{G} 0$ and a diminished $\mathrm{S} / \mathrm{G} 2$ population (Figure 1d). These findings suggested that cell cycle exit was the reason for our unsuccessful attempts of SATB1 expression in MEFs.

Immortalization of MEFs correlates with acquired mutations in growth control genes including the p53 or retinoblastoma (RB) pathways. ${ }^{27,28}$ To characterize expression changes in SATB1infected immortalized MEFs, we performed western analysis (Figure 1e). Whereas p53 and p27 protein levels were unchanged from controls, a strong increase in p16 and the RB-family protein p130 was clearly evident in SATB1-expressing cells. Increased p130 expression has previously been implicated in growth arrest. ${ }^{29,30}$ Furthermore, we observed a reduction of E2F1 and increase of cyclin D2, which is also consistent with entry into senescence. ${ }^{31,32}$ Thus, SATB1 expression led to an increased expression of p16 and changes in RB-family proteins whereas p53 was unchanged.
Genetic requirements for SATB1-induced senescence

p16 is a crucial factor for induction of senescence and can be activated through oncogenic signals. ${ }^{31,33,34}$ We therefore investigated whether a mutation of p16 could overcome the SATB1-induced proliferation arrest. We infected p16-deficient (p16-/-) primary MEFs with SATB1 virus. In contrast to wild-type MEFs, SATB1 expression did not cause growth inhibition in p16-/- MEFs. Proliferation was comparable between SATB1- and control virus-infected p16-/- MEFs (Figure 2a), and no change in senescence markers (Figures $2 \mathrm{~b}$ and c) or cell cycle distribution (Figure 2d) was observed. Western analysis showed only minor changes, following SATB1 expression in p16-/ - MEFs (Figure 2e). The observation that in the absence of p16 SATB1 expression was tolerated is consistent with the idea that SATB1 overexpression is not toxic, but triggers cell cycle arrest in wild-type MEFs.

We next tested a potential involvement of the p53 pathway in SATB1-induced senescence. For this we expressed SATB1 in MEFs isolated from p53-/- mice. These MEFs are immortal and do not enter crisis in culture. Infection of primary p53-/ - MEFs with SATB1 virus yielded SATB1-expressing colonies, albeit, at a reduced rate compared with control virus-infected cultures (Supplementary Figure 1, and data not shown). SATB1-expressing p53 - / - MEFs showed reduced proliferation (Supplementary Figure 1a), increased $\beta$-galactosidase activity (Supplementary Figures $1 \mathrm{~b}$ and $\mathrm{c}$ ) and a relative increase of G0/G1 and G2/M populations at the expense of S-phase cells (Supplementary Figure 1d). Western analysis detected increased expression of p16 and p130 compared with empty vector-infected controls (Supplementary Figure 1e). In addition, we noticed a decrease in p27 and a reduction of E2F1 expression. These observations indicate that deletion of p53 did not prevent SATB1-induced G1 arrest in MEFs to the same extent as deletion of $p 16$.

A critical downstream target of p16 is the RB pathway. We therefore investigated the effect of SATB1 expression in primary

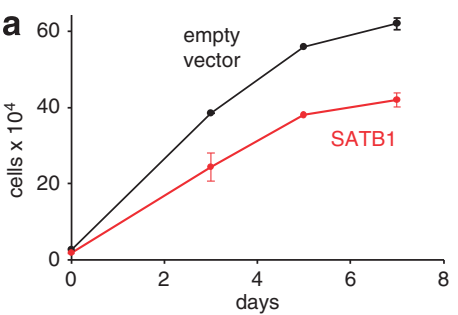

b

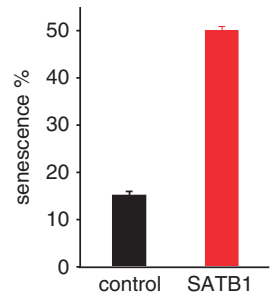

C

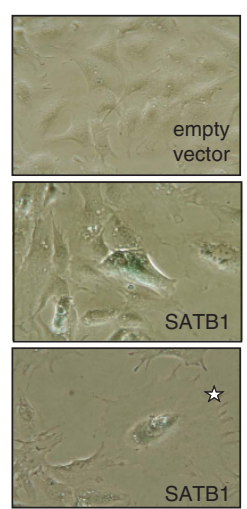

d

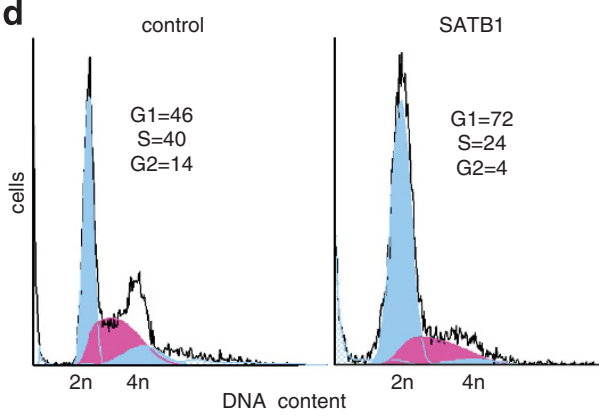

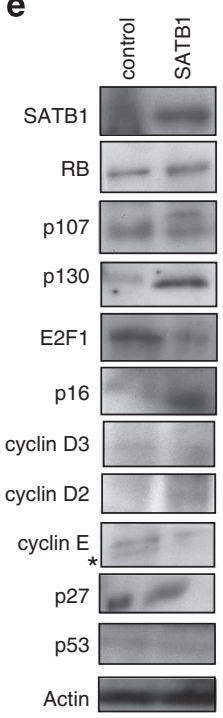

Figure 1. SATB1 induces senescence in MEFs. (a) Cumulative cell numbers of SATB1 virus (red) and empty vector-infected MEFs (black) are plotted. (b) Percentage of senescent cells identified by $\beta$-galactosidase staining in SATB1-infected (red) compared with control-infected cultures (black). (c) Representative images of SATB1 and control-infected MEFs. SATB1-expressing MEFs show a characteristic flat morphology (star). (d) Cell cycle analysis of control- and SATB1-infected MEFs. (e) Western analysis of SATB1- and control-infected MEFs. Actin was used to control for loading. The asterisk marks a senescent cell. 

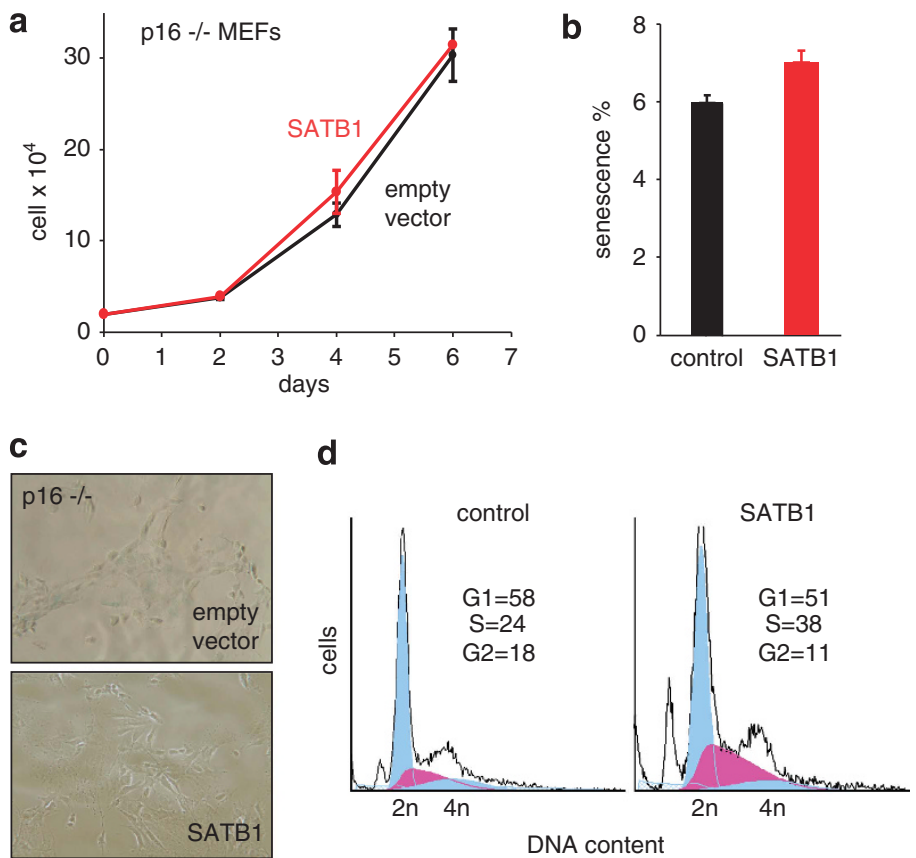

Figure 2. SATB1 expression does not induce senescence in p16-/ - MEFs. (a) Cumulative cell numbers of SATB1- (red) and control (black)infected p16-/- MEFs. (b) Percentage of senescent cells in SATB1- (red) compared with control-infected p16-/- MEFs (black). (c) Representative image of $\beta$-galactosidase stained (blue) SATB1- and control-infected p16-/ - MEFs shows that both are negative. (d) Cell cycle analysis of control-infected and SATB1-infected p16-/ - MEFs. (e) Western analysis of SATB1- and control-infected p16 - / - MEFs. Actin was used to control for loading.

MEFs isolated from $\mathrm{RB}-/$ - mice. Similar to our experiments in wild-type MEFs, we did not obtain colonies after infection with SATB1 expression virus and puromycin selection. SATB1 expression in immortalized $\mathrm{RB}-/-\mathrm{MEFs}$ reduced growth (Supplementary Figure 2a), increased senescence markers (Supplementary Figures $2 \mathrm{~b}$ and $\mathrm{c}$ ) and led to an increase in G1 and decrease in S/G2 cells (Supplementary Figure 2d). Western analysis showed a marked increase of p16, cyclin D2 and p130, as well as a reduction in E2F1 and p27 (Supplementary Figure 2e). The majority of these cells showed SATB1 expression (Supplementary Figure 2f). These results suggested that loss of RB was not sufficient to overcome an SATB1-induced cell cycle exit (Supplementary Figure 2g).

To address whether other members of the RB family compensate for the loss of $\mathrm{RB}$, we investigated the effect of SATB1 expression in MEFs isolated from RB - / - p107-/ p130 - / - triple-mutant (TM) mice. TM MEFs have the characteristic of being immortal because of complete loss of the RB pathway. SATB1-infected TM MEFs grew slower than control empty vector-infected TM MEFs (Figure 3a). Cell cycle analysis showed that SATB1 led to a large increase in G2/M cells (Figure $3 b$ ). We did not detect changes in $\beta$-galactosidase activity but a high fraction of apoptotic cells in SATB1-expressing cultures using terminal deoxynucleotidyl transferase dUTP nick-end labeling analysis (Figures $3 c$ and d). These observations are consistent with a previously reported G2/M arrest of TM MEFs in response to cytostatic signals. ${ }^{35}$ We further detected increased number of $\gamma-\mathrm{H} 2 \mathrm{AX}$ foci indicative of DNA damage in SATB1-expressing TM MEFs compared with control cells (Figures $3 i$ and $j$ ), suggesting that disruption of G1 cell cycle control has led to genomic damage. Western analysis further showed that SATB1 expression led to increased p16 and E2F1 protein levels independent of RB-family proteins (Figure 3g).

In order to establish that these changes were indeed attributable to loss of the RB pathway, we restored RB function using a viral vector in SATB1-expressing TM MEFs. SATB1-expressing TM MEFs infected with RB virus had a markedly higher fraction of senescent cells compared with TM MEFs without SATB1 (Figure 3e). Importantly, the G2/M arrest in SATB1-expressing TM MEFs was rescued after RB expression, and a pronounced G0/G1 population was observed (Figure 3f). Expression of SATB1 and RB was observed by immunofluorescence analysis and western analysis (Figure $3 \mathrm{~h}$ ). Taken together, these observations demonstrate that restoration of RB function was sufficient for SATB1-induced G0/G1 arrest and senescence in TM MEFs, indicating a critical role for p16 and the RB pathways in SATB1-induced cell cycle exit.

SATB1 interacts with RB/E2F1 complexes in gene regulation

To further investigate SATB1 expression and RB/E2F complexes, we performed immunofluorescence analysis on a panel of human cell lines that have previously been reported to express SATB1. These included MRC-5 embryonic lung human fibroblasts, the Jurkat acute T-cell leukemia cell line and the K562 erythroleukemia cell line. In all these cells RB and other RB-family proteins were expressed. In some of the cells characteristic nuclear patterns were observed whereby significant overlap with SATB1 was evident (Supplementary Figure 3a-e). To investigate a potential biochemical interaction further, we expressed human influenza haemagglutinin(HA)--tagged RB (RB-HA) and $3 \times$ Flag-tagged SATB1 in Hela cells and human embryonic kidney HEK-293T cells (Figures $4 a$ and $b$, and Supplementary Figure 3f). Both RB-HA and E2F1-HA were immunoprecipitated by Flag-tagged SATB1 (Figures 4a and b). The reciprocal interactions were also detected with HA-tagged E2F1 and RB, showing that SATB1 can interact with RB/E2F1 complexes.

We further investigated a series of previously characterized RB mutations that differ in their ability to form complexes with E2F1. ${ }^{36}$ We observed an interaction between SATB1- and RB-derived proteins that can bind to E2F1 ( $\Delta 685$ and $\Delta 657)$, but not with RB mutants $(\Delta 663$ or $\Delta 651)$ that form an unstable complex with E2F1 (Figures 4c and d). Therefore, the interaction of 
a

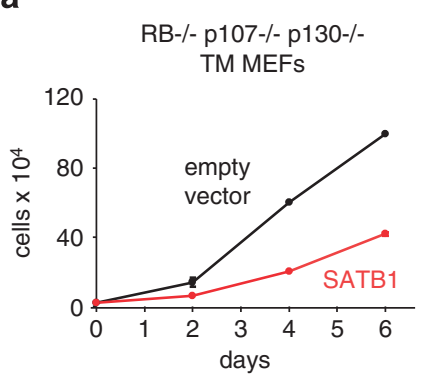

c

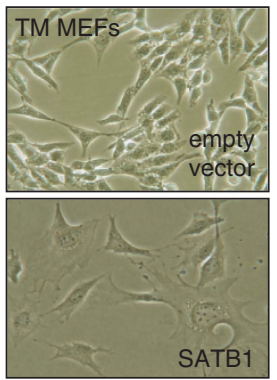

d

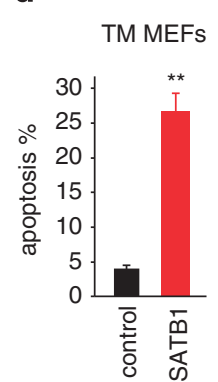

e

Restoration of RB in TM MEFs

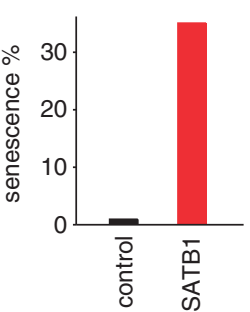

b

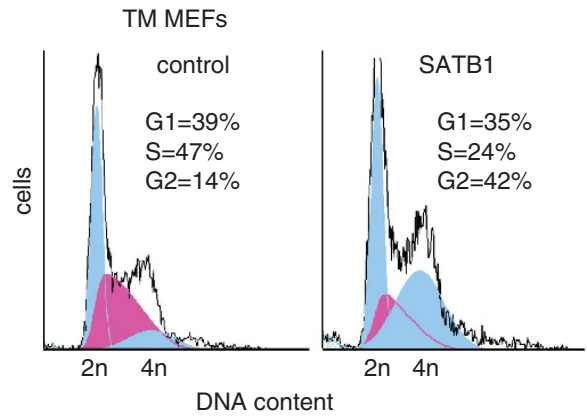

f

TM MEFs transfected with RB
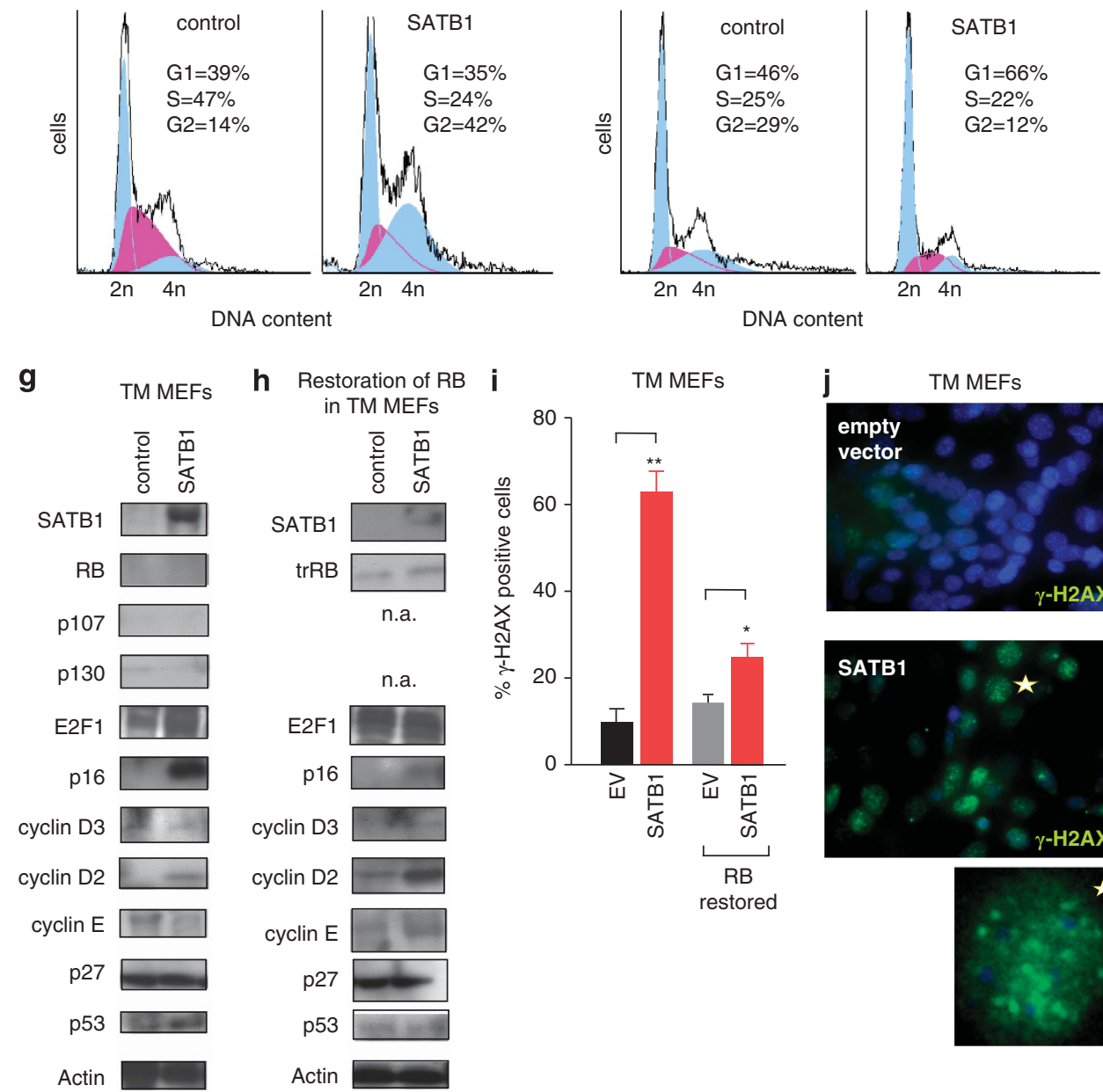

h Restoration of RB i in TM MEFs

\section{i}

TM MEFs
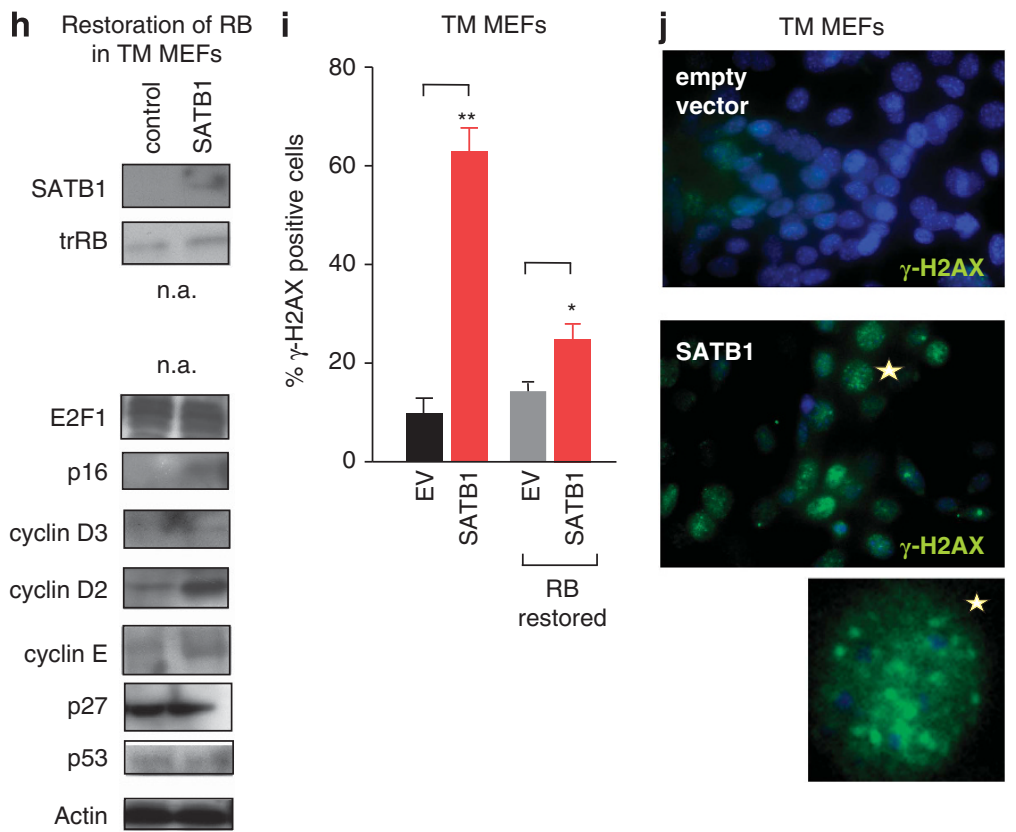

Figure 3. Rescue of SATB1-induced G0/G1 arrest by combined deletion of RB, p107 and p130. (a) Cumulative cell numbers of SATB1- (red) and control-infected (black) TM MEFs. (b) Cell cycle profile showing that SATB1-infected TM MEFs arrest in G2/M. (c) Representative image of $\beta$-galactosidase stained (blue) SATB1- and control-infected TM MEFs. Both are negative for $\beta$-galactosidase but SATB1-infected TM MEFs have a senescentoid phenotype. (d) Apoptosis was quantified by TUNEL assay. Error bars represent s.d. $(n=9)$. Statistical testing was performed using Student's $t$-test and a $P$-value of ${ }^{* *} P<0.001$ was calculated. (e) Quantification of senescence observed by $\beta$-galactosidase activity and (f) cell cycle profile after restoration of RB function in TM MEFs. Control empty vector-infected and SATB1-infected TM MEFs were infected with RB expression virus, and cell cycle profiles and senescence was determined. (g) Western analysis of SATB1- and control-infected TM MEFs. Actin was used to control for loading. (h) Western analysis of SATB1- and control-infected TM MEFs after infection with RB virus (n.a., not analyzed). (i) Statistical representation of $\gamma-\mathrm{H} 2 \mathrm{AX}$ foci in TM MEFs transfected with SATB1 expression or control virus (EV). Also, data after restoration of RB expression in TM MEFs is shown. Error bars represent s.d. $(n=9)$; ${ }^{*} P<0.05 ;{ }^{* *} P<0.01$. $P$-values were determined by the Student's $t$-test. (j) Representative images showing $\gamma-\mathrm{H} 2 \mathrm{AX}$ foci in TM MEFs transfected with EV.

SATB1 and RB appeared to depend on the formation of an $\mathrm{RB} / \mathrm{E} 2 \mathrm{~F} 1$ complex. Lastly, we investigated whether an interaction between SATB1 and E2F1 could be observed independent of RB. For this we used mouse embryonic stem cells in which the interaction between E2F1 and RB is eliminated by constitutive hyperphosphorylation of $\mathrm{RB}^{37,38}$ and SATB1 is expressed. ${ }^{5,8}$
Immunoprecipitation with antisera to E2F1 co-precipitated endogenous SATB1 in embryonic stem cell extracts (Figure 4e). Conversely, E2F1 was co-immunoprecipitated by the SATB1 antiserum (Figure 4e). These data indicate that endogenous SATB1 can interact with E2F1, independent of RB in mouse embryonic stem cells. We were not able to detect an interaction 


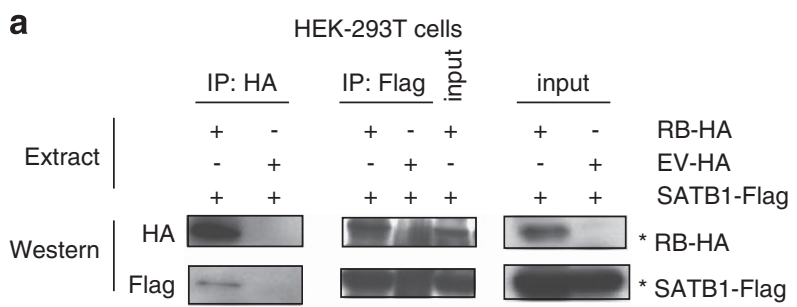

b

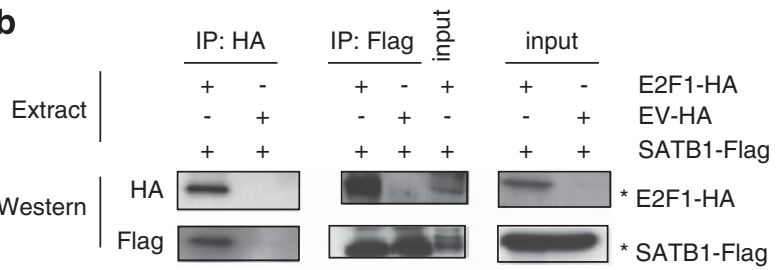

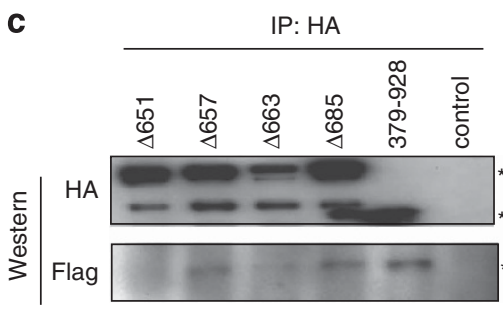

d

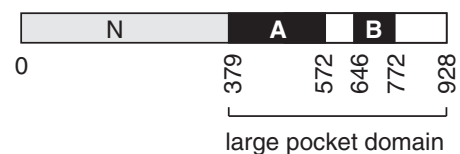

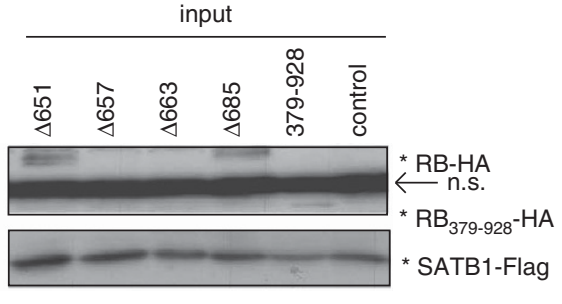

e

ES cells

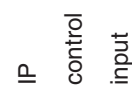

IP: E2F1 $\square$ * SATB1

IP: SATB1
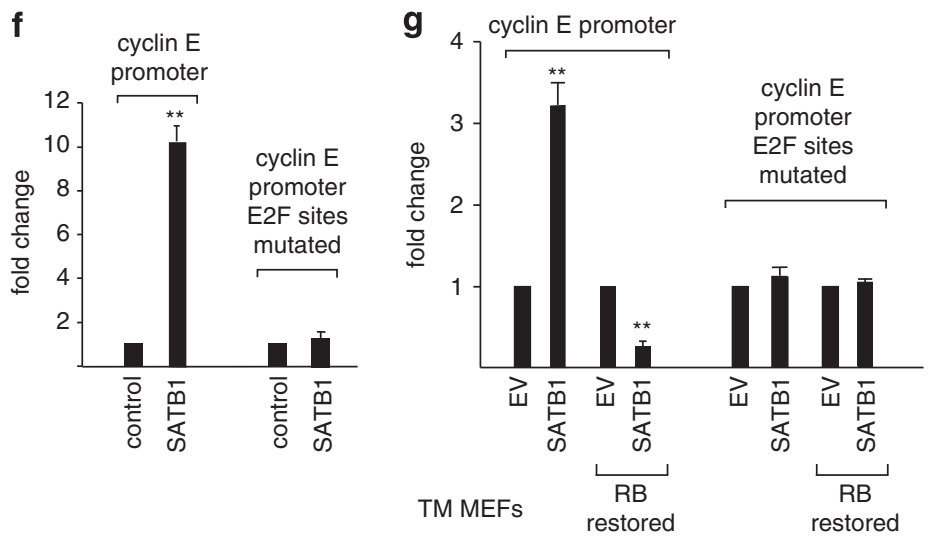

Figure 4. SATB1 interacts with RB and E2F1. (a) Coimmunoprecipitation of RB and SATB1 in lysates from HEK-293T cells transfected with 3xFlag-SATB1 and either RB-HA or HA empty vector (EV-HA). (b) Coimmunoprecipitation of E2F1 and SATB1 in lysates from HEK-293T cells transfected with 3xFlag-SATB1 and either E2F1-HA or EV-HA. (c) Coimmunoprecipitation of a panel of RB mutants deficient and proficient for forming stable complexes with E2F1 (see text) in lysates from HEK-293T cells transfected with 3xFlag-SATB1, together with RB-HA mutants or EV-HA. Note: the band indicated by an arrow is nonspecific (n.s.). (d) A scheme of RB protein domains showing amino-acid positions and protein domains. (e) Coimmunoprecipitation of endogenous E2F1 and SATB1 in nuclear lysates from undifferentiated mouse embryonic stem (ES) cells. (f) Cyclin E promoter activity was determined by dual-luciferase assay in $24 \mathrm{~h}$ after transfection, and is plotted as the ratio of the relative luciferase units. Results are expressed as fold increase in SATB1-transfected normalized to the control cells. Error bars represent s.d. $(n=9)$. Statistical significance was determined by the Student's $t$-test $\left({ }^{* *} P<0.01\right)$. (g) Cyclin E promoter activity in SATB1-expressing TM MEFs either expressing RB or not as in (f). Asterisks indicate band localization.

between SATB1 and E2F1 in immunoprecipitates from p16-deficient SATB1-expressing MEFs despite repeated attempts, which is likely attributable to the lower amount of E2F1 and SATB1 protein in these cells.
To further investigate the relevance of an interaction between SATB1 and E2F1 for gene expression, we examined the cyclin E promoter that is a well-established E2F1 target. For this we used constructs containing a luciferase reporter under the control of 
either the wild-type cyclin E promoter or a mutated control, in which the E2F-binding sites were removed. ${ }^{39}$ Co-transfection of SATB1 increased transcriptional activity of the wild-type cyclin E promoter 10-fold in HeLa cells (Figure 4f). In contrast, SATB1 had no effect when the E2F-binding sites were mutated (Figure 4f). Activation of the cyclin E promoter was also found in SATB1expressing TM MEFs (Figure 4g). Importantly, SATB1 expression resulted in a 3.5 -fold reduction in transcriptional activity of the wild-type cyclin E promoter in TM MEFs after restoration of RB expression, but SATB1 had no effect on the cylcin E promoter when the E2F sites were mutated (Figure 4g). These findings suggest that SATB1 orchestrates gene expression changes in combination with E2F on target genes.

SATB1 induces anchorage-independent growth and transformation in p16-/ - MEFs

We next investigated the phenotype of SATB1-expressing cells. For this we performed in vitro assays for cellular transformation using p16-deficient MEFs that either virally express SATB1 or controls that were infected by empty vector. SATB1-expressing p16-/- MEFs formed colonies in soft agar whereas no colonies were detected in controls (Figure $5 \mathrm{a}$ ). This observation indicates that SATB1 expression induced the capacity of anchorage-independent growth of p16-/- MEFs. To further assess migratory properties, we measured directed migration into an artificial 'wound' that was made in a confluent monolayer culture. SATB1 expression significantly enhanced migration and wound closure after $12 \mathrm{~h}$ in p16 - / - MEFs (Figure 5b). Increased cell motility is further consistent with the observation of actin reorganization and an increase in focal adhesions in SATB1expressing cells (Figure $5 \mathrm{c}$ ). For investigating invasiveness, we performed BD Matrigel Invasion Chamber assays (BD Biosciences, San Jose, CA, USA). These chambers consist of a membrane with $8-\mathrm{mm}$ pores that is coated with a $30-\mathrm{mm}$ layer of extracellular matrix. SATB1 expression led to a fivefold increase in the number of cells that migrate through this matrix layer, indicating that SATB1 elicited invasive properties in this assay (Figure $5 \mathrm{~d}$ ). Taken together, our results demonstrate that in the absence of p16 SATB1 leads to transformation and induces motility and invasiveness in MEFs, consistent with finding in human tumors.

\section{DISCUSSION}

Our study establishes an in vitro model for investigating the role of SATB1 in tumorigenesis. We find that untransformed cells arrest upon heterologous SATB1 expression. In contrast, p16-deficient cells are reprogrammed by SATB1 expression to a motile and invasive phenotype. This finding suggests that SATB1 can have a differential effect on cell proliferation. Our observations of anchorage-independent growth potential further support the idea that SATB1 has transforming potential. This is consistent with the observed role of SATB1 in tumor progression. Our study also indicates that SATB1 interacts with E2F in regulating the cyclin E promoter. This finding might explain some of the gene expression a
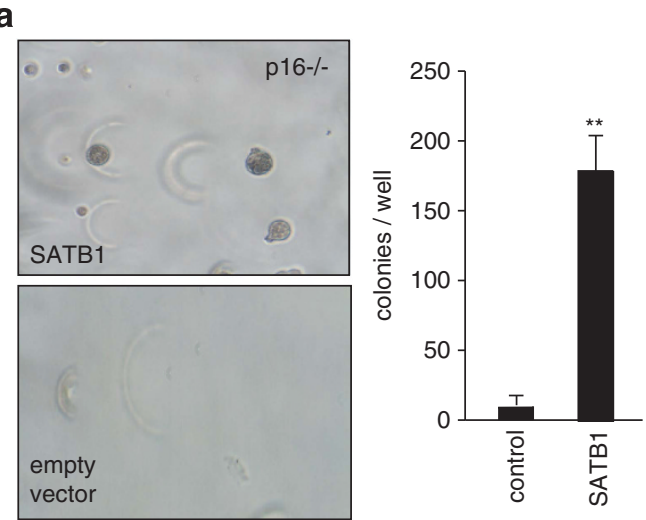

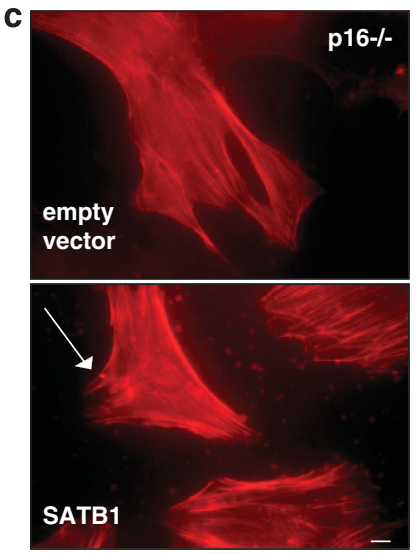

b
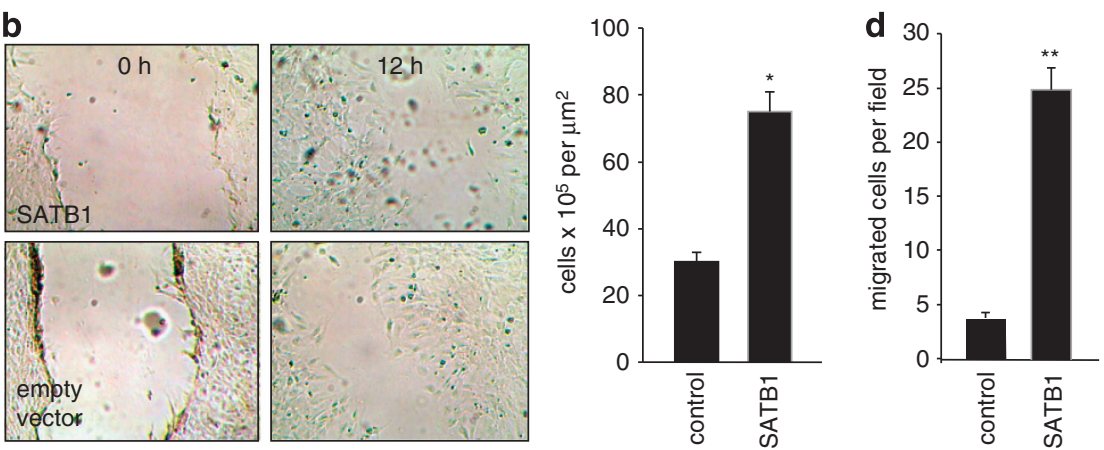

Figure 5. SATB1 expression leads to transformation in the absence of $p 16$. (a) SATB1-expressing p16 - / - MEFs form colonies in soft agar in p16 - / - MEFS. The number of colonies per well were counted and plotted. Error bars represent s.d. $\left(n=9 ;{ }^{* *} P<0.01\right)$. (b) Wounding assay of confluent cell layers of SATB1-expressing and control p16-/ - MEFs. The number of cells that migrated into a delimited wound area after $12 \mathrm{~h}$ is plotted. Cells in three defined area per group per experiment were quantified in three independent experiments with three technical replications $\left({ }^{*} P<0.05\right)$. (c) Representative image of phalloidin stained cells showing actin cytoskeleton reorganization in SATB1-expressing p16-/ - MEFs. Focal adhesion site is indicated by arrow. Scale bars represents $10 \mu \mathrm{m}$. (d) Quantification of Matrigel chamber migration assay of SATB1-expressing and control p16-/ - MEFs. Error bars represent s.d. $(n=9)$. Statistical significance was assessed by the Student's $t$-test; $* * 0<0.01$. 
changes and alterations of cellular phenotype associated with SATB1 expression. Our results in TM MEFs suggest that SATB1 can activate gene expression in the absence of RB but represses the cyclin $\mathrm{E}$ promoter when RB expression is restored. In the absence of RB-family proteins SATB1 induces a G2/M arrest and apoptosis. We also find an increased number of $\gamma-\mathrm{H} 2 \mathrm{AX}$ foci associated with the G2/M arrest of SATB1-expressing TM MEFs. This suggests that SATB1 induces an uncontrolled entry into cell division, when the G1 checkpoint is compromised, leading to genomic damage and apoptosis.

p16 appears to be a major gatekeeper of transformation in our study. We find upregulation of p16 upon SATB1 expression is independent of the RB family of proteins and of p53 and, thus, could be a direct consequence of SATB1 expression. In the absence of p16 SATB1 and E2F might cooperate to promote growth and invasiveness. This raises the question whether p16 mutations are more generally associated with SATB1 expression in human cell lines. Human cell lines that express SATB1, including K562 human erythroleukemia cells, Jurkat T cells, MRC-5 and WI-38 fibroblasts, show either very low expression of p16 or a loss of p16 due to a homozygous mutation. Re-expression of p16 in K562 and Jurkat cells has been shown to induce a growth arrest. ${ }^{40}$ Importantly, the SATB1-expressing Hs-578T and MDA-MB-231 breast cancer cell lines carry homozygous deletions of $\mathrm{p} 16,{ }^{16}$ and restoration of $\mathrm{p} 16$ expression in MDA-MB-231 breast cancer cells leads to growth arrest. ${ }^{41}$ These findings show that p16 deletions are frequently associated with SATB1 expression in human cells and tumors. We have, thus far, not been able to identify a cell line that expresses SATB1 and has intact p16 expression from the literature. Thus, genetic loss of p16 might be a contributing factor for transformation by SATB1.

An interesting result is that the deletion of p16 and of the RB family of proteins does not lead to equivalent outcomes after SATB1 expression. Residual activity of RB proteins in p16-deficient MEFs or additional functions of p16 might explain the observed differences.

A recent report has also observed a differential response of several human breast cancer cell lines to SATB1 expression, further supporting the idea that the genetic makeup of cells critically determines SATB1 function. ${ }^{42}$ Our results suggest that the intersection of SATB1 with the p16 and RB/E2F pathways is an important aspect of the progression of SATB1-positive cancers. Considering the p16 status of SATB1 positive tumors could have potential implication for diagnosis and therapy.

\section{MATERIALS AND METHODS}

Cell culture and transfections

K562, WI-38, MRC-5, HEK-293T and Jurkat were obtained from American Type Culture Collection (ATCC), Manassas, VA, USA, and cultured as recommended. MEFs were cultured and immortalized as described previously. ${ }^{8}$ p16-/ - MEFS and matched controls were kindly provided by Hartmut Beug (Institute of Molecular Pathology (IMP), Vienna). TM (p105-/- p107-/- p130-/-) MEFs, RB - / - MEFs and matched controls were kindly provided by Roland Foisner (Max F Perutz Laboratories (MFPL), Vienna, Austria) with permission from Tyler Jacks (Massachusetts Institute of Technology, USA). p53-/- MEFs and matched controls were derived from p53-deficient embryos.

\section{Senescence and apoptosis analysis}

$\beta$-gal staining was performed using the Senescence $\beta$-Galactosidase Staining Kit (Number 9860, Cell Signaling Technology, Danvers, MA, USA). Terminal deoxynucleotidyl transferase dUTP nick-end labeling staining was performed using the in situ cell death detection kit (Roche Diagnostics, Mannheim, Germany).

Detection of DNA damage by $\gamma-\mathrm{H} 2 \mathrm{AX}$ staining

Samples were stained with primary mouse monoclonal anti- $\gamma-\mathrm{H} 2 \mathrm{AX}$ antibody H2A.X (phospho S139; ab2893, Abcam, Cambridge, UK), followed by secondary Alexa-488-conjugated anti-mouse IgG (Molecular Probes, Eugene, OR, USA). Nuclei were counterstained with DAPI (4,6-diamidino-2phenylindole-dihydrochroride). Three independent experiments $\left(n_{1}=3\right)$, with three replications per experiment $\left(n_{2}=9\right)$ were performed. Three hundred nuclei were counted per experiment. All $P$-values were determined by the Student's $t$-test.

\section{Cloning and plasmids}

Plasmids pSG5L HA RB (379-928), 496 pSG5L HA RB $\Delta 651$ (NAAIRS), 498 pSG5L HA RB $\triangle 663$ (NAAIRS), 500 pSG5L HA RB $\Delta 685$ (NAAIRS), 408 pSG5L HA E2F1, pSG5L HA RB, 432 pSG5L HA RB $\triangle 657$ were as published. ${ }^{36}$ PSGL5 was used as control. SATB1 (IRAUp969H0715D cDNA Clone (Imagenes, Berlin, Germany) was cloned into the pBabe-puro-internal ribosomal entry site-enhanced GFP vector. GFP-RB-FL was previously published, ${ }^{43}$ and pCMV10-3XFLAG-SATB1 was a gift from Sanjeev Galande (Indian Institute of Science Education and Research, Pune, India).

\section{Transfections, western blotting and immunoprecipitation}

Total cell extracts were prepared with radioimmunoprecipitation assay buffer. ${ }^{44}$ Nuclear cell extracts and western blots were performed as described.$^{44}$ Antibodies were used as follows: p16 1:300 (p16(C-20), rabbit anti-p16, Santa Cruz Biotechnology, Inc., Santa Cruz, CA, USA), cyclin D2 (cyclin D2 (M20), rabbit anti-cyclin D2, Santa Cruz), cyclin D3 1:1000 (cyclin D3 (C16), rabbit anti-cyclin D3, Santa Cruz), cyclin E 1:1000 (cyclin E (M20) rabbit anti-cyclin E, Santa Cruz), p27 1:300 (p27 (C-19), rabbit anti-p27, Santa Cruz), p53 1:500 (ab2433, rabbit anti-p53, Abcam), anti-SATB1 1:2000, ${ }^{2}$ E2F1 1:1000 (rabbit anti-E2F1 (H-137); Santa Cruz), RB 1:1000 (rabbit anti-RB (C-15), Santa Cruz), RB (phospho S608) antibody 1:1000 (ab60025, rabbit anti-RB (phospho S608; Abcam), p107 1:500 (rabbit antip107 (C-18), Santa Cruz), p130 1:500 (rabbit anti-p130 (C-20), Santa Cruz).

For immunoprecipitation experiments HEK-293T cells were transfected using Effectene (Qiagen, Hilden, Germany), with either PCMV10-3X FlagSATB1 and PSG5L HA RB or PCMV10-3X Flag-SATB1 and PCMV3-HA-E2F1. Cells transfected with PCMV10-3XFLAG-SATB1 and PCMV3-HA were used as a control. For analyzing RB mutants, transfections with PCMV10-3X FlagSATB1 and mutants described above ${ }^{36}$ were performed. Total cell extracts were prepared using radioimmunoprecipitation assay buffer $72 \mathrm{~h}$ after transfection. HA-immunoprecitations were performed using the Mammalian HA Tag IP/Co-IP Kit (Pierce-Thermo Scientific, Rockford, IL, USA). FLAG immunoprecipitation was performed using $A / G$ beads (SC 2003, Santa (ruz). Precipitates were analyzed by western blot using HA 1:1000 and FLAG 1:1000 antibodies (kindly provided by Jan Michael Peters, IMP, Vienna). Embryonic stem whole cell extracts were immunoprecipitated with A/G beads (SC 2003, Santa Cruz) using 1-2 $\mu \mathrm{g}$ SATB1 antibody (ab70004, rabbit anti-SATB1, Abcam) per $100-500 \mu \mathrm{g}$ of total protein and $1-2 \mu \mathrm{g}$ E2F1 antibody (rabbit anti-E2F1 (H-137), Santa Cruz) per $100-500 \mu \mathrm{g}$ of total protein. Western analysis was performed at least three times for three independent experiments.

Virus production and infection of MEFs

The human SATB1 CDNA (Imagenes) was inserted into the pBabe-puro-GFP vectors. Retrovirus production was performed as described. ${ }^{8}$ Briefly, producer Plat-E cells ${ }^{45}$ were transfected using Effectene (Qiagen). Viral supernatants were collected $48 \mathrm{~h}$ later and used for infection. MEFs were infected for 48 and $24 \mathrm{~h}$ later, selected with puromycin until colonies appeared. Colonies positive for GFP were picked using cloning cylinders (Millipore, Billerica, MA, USA) and expanded. For kinetics studies, $10^{4}$ cells were plated in six-well plates in triplicates and cell numbers were determined every 2 days using a CASY cell counter (Roche Diagnostics). For restoring RB activity SATB1- or EV-infected TM MEFs were transfected with GFP-RB-FL and selected for 3 weeks with G418. Three independent experiments were performed in triplicate $(n=9)$ using MEFs at less than eight passages, error bars represent s.d.

\section{Cell cycle analysis}

DNA content analysis was performed using propidium iodide/RNase buffer (BD Biosciences, San Jose, CA, USA). Briefly, $2 \times 10^{5}$ cells were harvested, fixed with $70 \%$ ethanol for $20 \mathrm{~min}$ on ice, washed and resuspended in $500 \mu \mathrm{l}$ of propidium iodide/RNase buffer, followed by analysis on flow cytometer (FACS calibur, BD Biosciences). Cell cycle profiles were analyzed using the multicycle software (Phoenix Flow Systems, San Diego, CA, USA). Presented results are representative of three independent experiments performed in triplicate $(n=9)$. 
Immunofluorescence analysis

WI-38, MRC-5, Hela cell lines and MEFS were grown on Roboz slides (Cell Point Scientific, Gaithersburg, MD, USA). K562 and Jurkat cell line cells were attached to adhesion slides (Marilienfeld, Lauda-Königshofen, Germany). Immunostaining was performed as described ${ }^{44}$ Briefly, cells were fixed for $10 \mathrm{~min}$ in $4 \%$ paraformaldehyde in phosphate-buffered saline (PBS), permeabilized for $5 \mathrm{~min}$ in $0.1 \% \mathrm{Na}$-citrate/0.5\% Triton X-100 and blocked for $30 \mathrm{~min}$ in PBS containing $5 \%$ bovine serum albumin and $0.1 \%$ Tween-20. Antibodies and dilutions used for the analysis were E2F1 1:100 (rabbit anti-E2F1 (H-137), Santa Cruz), RB 1:200 (rabbit anti-RB (C-15), Santa Cruz), underphosphorylated RB 1:300 (RB aa 514-610, mouse antihuman underphosphorylated RB, BD Biosciences), RB (phospho S608) antibody 1:200 (ab60025 rabbit anti-RB (phospho S608), Abcam), p107 1:100 (rabbit anti-p107(C-18), Santa Cruz), p130 1:100 (rabbit anti-p130 (C-20), Santa Cruz). SATB1 1:100 (ab49061 rabbit anti-SATB1, Abcam) and SATB1 1:300 (611182, mouse anti-human SATB1, BD Biosciences). Secondary antibodies were previously described. ${ }^{44}$ Vectashield (Vector Laboratories, Burlingame, CA, USA) was used as imaging medium. DNA was stained with DAPI (40,60-diamidino-2-phenylindole). Images were acquired at room temperature using a fluorescence microscope (Axioplan, Zeiss, Jena, Germany) equipped with a CCD camera (CoolSNAP fx, Photometrics, Tucson, AZ, USA), and analyzed using the MetaMorph image analysis software (Molecular Devices Corporation, Sunnyvale, CA, USA). Color levels were adjusted in Photoshop 7.0 (Adobe). For deconvolution microscopy, series of $0.2-\mathrm{mm}$ z-sections were collected on a DeltaVision Spectris Restoration Microscopy System (Applied Precision Inc., Issaquah, WA, USA) using a $100 \times 1.4 \mathrm{NA}$ planachromat objective, and subsequently deconvolution was performed using the proprietary SoftWorx algorithm (Applied Precision).

\section{Colony-forming assay in soft agar}

Cells $\left(1.5 \times 10^{5}\right)$ were plated in $0.36 \%$ agar in Dulbecco's modified Eagle growth medium (soft agar medium) per $60-\mathrm{mm}$ dish on top of a $0.72 \%$ hard agar layer. Cultures were reefed by adding $3 \mathrm{ml}$ soft agar medium after 1 and 2 weeks. After 21 days colonies were counted using an inverted microscope. Results represent three independent experiments that were performed in triplicate $(n=9)$.

\section{Wound healing assays}

An artificial wound was generated with a $10-\mu$ l pipette tip on confluent monolayers of $\mathrm{p} 16-/-\mathrm{SATB} 1$ and $\mathrm{p} 16-/$ - control empty vector MEFs grown in six-well culture plates in serum-containing medium. Photographs were taken at $0 \mathrm{~h}$ and $12 \mathrm{~h}$, respectively. Analysis of wound closure was calculated by counting the cells per $\mu \mathrm{m}^{2}$ of wound area at $12 \mathrm{~h}$. Results represent three independent experiments that were performed in triplicate $(n=9)$.

\section{Phalloidin staining}

For analyzing actin filaments, cells were incubated with Texas Red-X phalloidin (Molecular Probes), according to the manufacturer's protocol. Briefly, cells were fixed for $10 \mathrm{~min}$ in $4 \%$ paraformaldehyde in PBS permeabilized in Na-citrate/0.5\% Triton X-100, blocked for 30 min with $1 \%$ bovine serum albumion in PBS and incubated with Texas $\operatorname{Red}^{-} X$ phalloidin (1:100) dilution for $30 \mathrm{~min}$. 4',6-diamidino-2-phenylindole-dihydrochloride was used to stain DNA.

\section{Two-chamber migration and invasion assays}

Cell invasion was determined by using the BD Biocoat Matrigel Invasion Chamber $(8 \mu \mathrm{m}$ pore size, BD Biosciences) invasion assay (membrane coated with a layer of Matrigel extracellular matrix proteins), according to the manufacturer's instructions. Cells $\left(2.5 \times 10^{4}\right)$ were seeded in serum-free medium into the upper chamber, and $10 \%$ fetal calf serum was used as a chemoattractant in the lower chamber. After $18 \mathrm{~h}$, cells in the upper chamber were carefully removed using cotton buds and cells at the bottom of the membrane were fixed in $4 \%$ paraformaldehyde in PBS and stained with crystal violet (0.2\%). Quantification was performed by counting the stained cells. Three independent experiments were performed in triplicate $(n=9)$.

\section{Luciferase assays}

HeLa cells $\left(10^{5}\right)$, TM-EV; TM-SATB1;TM-EV-RB or TM-SATB1-RB were transiently transfected in six-well dishes using EFFECTENE reagent (Qiagen), according to the manufacturer's instructions and harvested $24 \mathrm{~h}$ after transfection. Two cyclin $\mathrm{E}$ promoter-driven luciferase constructs (Addgene, Cambridge, MA, USA) were used, as published. ${ }^{39}$ For transfection, $1 \mu \mathrm{g}$ of reporter plasmid was combined with $0.98 \mu \mathrm{g}$ of either the PCMV10-3XFLAG-SATB1 construct or the control PCMV10 empty vector, and $20 \mathrm{ng}$ of pRL-SV40 Renilla luciferase vector as an internal reference. Luciferase activities were measured using the Dual-Luciferase Reporter Assay System (Promega, Fitchburg, WI, USA), and results were normalized to Renilla luciferase activity. The presented results correspond to three independent experiments $\left(n_{1}=3\right)$, with three replications per experiment $\left(n_{2}=9\right)$.

\section{CONFLICT OF INTEREST}

The authors declare no conflict of interest.

\section{ACKNOWLEDGEMENTS}

We thank M Saltik, MH Idarraga, G Stengl and P Pasierbek for technical assistance, V Bhaskara for helpful discussions, S Galande, JM Peters, H Beug and R Foisner for reagents and advice. This research was supported by the IMP, the Vienna Science and Technology Fund (WWTF), and the Austrian Science Fund (FWF). RA is supported by ANII (Program INNOVA URUGUAY-DCI-ALA/2007/19.040 URU-European Union). AW is supported by a Wellcome Trust Senior Research Fellowship (Grant reference 087530/ Z/08/A).

\section{REFERENCES}

1 de Belle I, Cai S, Kohwi-Shigematsu T. The genomic sequences bound to special AT-rich sequence-binding protein 1 (SATB1) in vivo in Jurkat T cells are tightly associated with the nuclear matrix at the bases of the chromatin loops. J Cell Biol 1998; 141: 335-348.

2 Dickinson LA, Joh T, Kohwi Y, Kohwi-Shigematsu T. A tissue-specific MAR/SAR DNA-binding protein with unusual binding site recognition. Cell 1992; 70: 631-645.

3 Alvarez JD, Yasui DH, Niida H, Joh T, Loh DY, Kohwi-Shigematsu T. The MARbinding protein SATB1 orchestrates temporal and spatial expression of multiple genes during T-cell development. Genes Dev 2000; 14: 521-535.

4 Asanoma K, Kubota K, Chakraborty D, Renaud SJ, Wake N, Fukushima K et al. SATB homeobox proteins regulate trophoblast stem cell renewal and differentiation. J Biol Chem 2012; 287: 2257-2268.

5 Savarese F, Davila A, Nechanitzky R, De La Rosa-Velazquez I, Pereira CF, Engelke R et al. Satb1 and Satb2 regulate embryonic stem cell differentiation and nanog expression. Genes Dev 2009; 23: 2625-2638.

6 Cai S, Lee CC, Kohwi-Shigematsu T. SATB1 packages densely looped, transcriptionally active chromatin for coordinated expression of cytokine genes. Nat Genet 2006; 38: 1278-1288.

7 Cai S, Han HJ, Kohwi-Shigematsu T. Tissue-specific nuclear architecture and gene expression regulated by SATB1. Nat Genet 2003; 34: 42-51.

8 Agrelo R, Souabni A, Novatchkova M, Haslinger C, Leeb M, Komnenovic V et al. SATB1 defines the developmental context for gene silencing by Xist in lymphoma and embryonic cells. Dev Cell 2009; 16: 507-516.

9 Yasui D, Miyano M, Cai S, Varga-Weisz P, Kohwi-Shigematsu T. SATB1 targets chromatin remodelling to regulate genes over long distances. Nature 2002; 419: 641-645.

10 Pavan Kumar P, Purbey PK, Sinha CK, Notani D, Limaye A, Jayani RS et al. Phosphorylation of SATB1, a global gene regulator, acts as a molecular switch regulating its transcriptional activity in vivo. Mol Cell 2006; 22: 231-243.

11 Purbey PK, Singh S, Notani D, Kumar PP, Limaye AS, Galande S. Acetylationdependent interaction of SATB1 and CtBP1 mediates transcriptional repression by SATB1. Mol Cell Biol 2009; 29: 1321-1337.

12 Tan JA, Sun Y, Song J, Chen Y, Krontiris TG, Durrin LK. SUMO conjugation to the matrix attachment region-binding protein, special AT-rich sequence-binding protein-1 (SATB1), targets SATB1 to promyelocytic nuclear bodies where it undergoes caspase cleavage. J Biol Chem 2008; 283: 18124-18134.

13 Notani D, Gottimukkala KP, Jayani RS, Limaye AS, Damle MV, Mehta S et al. Global regulator SATB1 recruits beta-catenin and regulates $T(H) 2$ differentiation in Wnt-dependent manner. PLoS Biol 2010; 8: e1000296.

14 Beyer M, Thabet Y, Muller RU, Sadlon T, Classen S, Lahl K et al. Repression of the genome organizer SATB1 in regulatory T cells is required for suppressive function and inhibition of effector differentiation. Nat Immunol 2011; 12: 898-907. 
15 Fessing MY, Mardaryev AN, Gdula MR, Sharov AA, Sharova TY, Rapisarda V et al. p63 regulates Satb1 to control tissue-specific chromatin remodeling during development of the epidermis. J Cell Biol 2011; 194: 825-839.

16 Han HJ, Russo J, Kohwi Y, Kohwi-Shigematsu T. SATB1 reprogrammes gene expression to promote breast tumour growth and metastasis. Nature 2008; 452 187-193.

17 Cheng C, Lu X, Wang G, Zheng L, Shu X, Zhu S et al. Expression of SATB1 and heparanase in gastric cancer and its relationship to clinicopathologic features. Apmis 2010; 118: 855-863.

18 Lu X, Cheng C, Zhu S, Yang Y, Zheng L, Wang G et al. SATB1 is an independent prognostic marker for gastric cancer in a Chinese population. Oncol Rep 2010; 24 981-987.

19 Zhao XD, Ji WY, Zhang W, He LX, Yang J, Liang HJ et al. Overexpression of SATB1 in laryngeal squamous cell carcinoma. ORL J Otorhinolaryngol Relat Spec 2010; 72 $1-5$.

20 Meng WJ, Yan H, Zhou B, Zhang W, Kong XH, Wang R et al. Correlation of SATB1 overexpression with the progression of human rectal cancer. Int J Colorectal Dis 2011; 27: 143-150.

21 Chen H, Takahara M, Oba J, Xie L, Chiba T, Takeuchi S et al. Clinicopathologic and prognostic significance of SATB1 in cutaneous malignant melanoma. J Dermatol Sci 2011; 64: 39-44.

22 Mclnnes N, Sadlon TJ, Brown CY, Pederson S, Beyer M, Schultze JL et al. FOXP3 and FOXP3-regulated microRNAs suppress SATB1 in breast cancer cells. Oncogene 2011; 31: 1045-1054.

23 Yamayoshi A, Yasuhara M, Galande S, Kobori A, Murakami A. Decoy-DNA against special AT-rich sequence binding protein 1 inhibits the growth and invasive ability of human breast cancer. Oligonucleotides 2011; 21: 115-121.

24 lorns E, Hnatyszyn HJ, Seo P, Clarke J, Ward T, Lippman M. The role of SATB1 in breast cancer pathogenesis. J Natl Cancer Inst 2010; 102: 1284-1296.

25 Kohwi-Shigematsu T, Han HJ, Russo J, Kohwi Y. Re: the role of SATB1 in breast cancer pathogenesis. J Natl Cancer Inst 2010; 102: 1879-1880.

26 Hanker LC, Karn T, Mavrova-Risteska L, Ruckhaberle E, Gaetje R, Holtrich U et al. SATB1 gene expression and breast cancer prognosis. Breast 2010; 20: 309-313.

27 Odell A, Askham J, Whibley C, Hollstein M. How to become immortal: let MEFs count the ways. Aging (Albany NY) 2010; 2: 160-165.

$28 \mathrm{Kim} \mathrm{H}$, You S, Farris J, Foster LK, Foster DN. Post-transcriptional inactivation of p53 in immortalized murine embryo fibroblast cells. Oncogene 2001; 20: $3306-3310$

29 Fiorentino FP, Symonds CE, Macaluso M, Giordano A. Senescence and p130/Rbl2 a new beginning to the end. Cell Res 2009; 19: 1044-1051.

30 Harb G, Vasavada RC, Cobrinik D, Stewart AF. The retinoblastoma protein and its homolog $\mathrm{p} 130$ regulate the G1/S transition in pancreatic beta-cells. Diabetes 2009; 58: $1852-1862$.

31 Alcorta DA, Xiong Y, Phelps D, Hannon G, Beach D, Barrett JC. Involvement of the cyclin-dependent kinase inhibitor p16 (INK4a) in replicative senescence of normal human fibroblasts. Proc Natl Acad Sci USA 1996; 93: 13742-13747.

32 Meyyappan M, Wong H, Hull C, Riabowol KT. Increased expression of cyclin D2 during multiple states of growth arrest in primary and established cells. Mol Cell Biol 1998; 18: 3163-3172.

33 Kim WY, Sharpless NE. The regulation of INK4/ARF in cancer and aging. Cell 2006; 127: $265-275$.

34 Takahashi A, Ohtani N, Hara E. Irreversibility of cellular senescence: dual roles of p16INK4a/Rb-pathway in cell cycle control. Cell Div 2007; 2: 10.

35 Sage J, Mulligan GJ, Attardi LD, Miller A, Chen S, Williams B et al. Targeted disruption of the three Rb-related genes leads to loss of $\mathrm{G}(1)$ control and immortalization. Genes Dev 2000; 14: 3037-3050.

36 Sellers WR, Novitch BG, Miyake S, Heith A, Otterson GA, Kaye FJ et al. Stable binding to $\mathrm{E} 2 \mathrm{~F}$ is not required for the retinoblastoma protein to activate transcription, promote differentiation, and suppress tumor cell growth. Genes Dev 1998; 12: 95-106.

37 Conklin JF, Sage J. Keeping an eye on retinoblastoma control of human embryonic stem cells. J Cell Biochem 2009; 108: 1023-1030.

38 White J, Dalton S. Cell cycle control of embryonic stem cells. Stem Cell Rev 2005; 1 : 131-138.

39 Geng Y, Eaton EN, Picon M, Roberts JM, Lundberg AS, Gifford A et al. Regulation of cyclin E transcription by E2Fs and retinoblastoma protein. Oncogene 1996; 12: $1173-1180$

40 Quesnel B, Preudhomme C, Lepelley P, Hetuin D, Vanrumbeke M, Bauters F et al. Transfer of p16inka/CDKN2 gene in leukaemic cell lines inhibits cell proliferation. Br J Haematol 1996; 95: 291-298.

41 Kim M, Katayose $Y$, Rojanala L, Shah S, Sgagias M, Jang $L$ et al. Induction of apoptosis in p16INK4A mutant cell lines by adenovirus-mediated overexpression of p16INK4A protein. Cell Death Differ 2000; 7: 706-711.

42 Ordinario E, Han HJ, Furuta S, Heiser LM, Jakkula LR, Rodier F et al. ATM suppresses SATB1-induced malignant progression in breast epithelial cells. PloS One 2012; 7: e51786.

43 Khan MZ, Brandimarti R, Shimizu S, Nicolai J, Crowe E, Meucci O. The chemokine CXCL12 promotes survival of postmitotic neurons by regulating Rb protein. Cell Death Differ 2008; 15: 1663-1672.

44 Leeb $M$, Wutz A. Ring1B is crucial for the regulation of developmental contro genes and PRC1 proteins but not X inactivation in embryonic cells. J Cell Biol 2007; 178: 219-229.

45 Morita S, Kojima T, Kitamura T. Plat-E: an efficient and stable system for transient packaging of retroviruses. Gene Therapy 2000; 7: 1063-1066.

(c) (D) This work is licensed under a Creative Commons AttributionSA NonCommercial-ShareAlike 3.0 Unported License. To view a copy of this license, visit http://creativecommons.org/licenses/by-nc-sa/3.0/

Supplementary Information accompanies this paper on the Oncogene website (http://www.nature.com/onc) 